\title{
LA TENDENCIA A QUEJARSE ANTE UN TERCERO TRAS UN FALLO EN EL SERVICIO: INFLUENCIA DE LA JUSTICIA INTERPERSONAL Y LA EXPERIENCIA PREVIA EN EL SECTOR HOTELERO ESPAÑOL
}

\author{
OSCAR AGUILAR-ROJAS \\ Universidad de Costa Rica, Costa Rica \\ Oscar.aguilarrojas@ucr.ac.cr \\ CARMINA FANDOS-HERRERA \\ Universidad de Zaragoza, España. \\ cfandos@unizar.es
}

\section{RESUMEN}

Nunca antes tantos servicios habían sido comprados, vendidos y consumidos como lo son hoy y además, nunca antes los consumidores habían tenido tantos medios para manifestar su insatisfacción a través de una queja. Este trabajo aborda el estudio de las quejas ante un tercero en el contexto de los hoteles teniendo en cuenta la experiencia previa del cliente y el trato del que ha sido objeto. El objetivo de este artículo era analizar qué influencia puede ejercer la justicia interpersonal, la experiencia previa, el género y la edad sobre la intención de quejarse ante un tercero. Además, se ha estudiado cuáles son los instrumentos de queja que utiliza un cliente insatisfecho tras un fallo en un servicio, si los periódicos, las redes sociales, las asociaciones de consumidores o acometer acciones legales. Se presentan resultados interesantes de los que se derivan consecuencias aplicables a una gestión efectiva de las quejas en el sector de los servicios y recomendaciones para evitar que se interpongan dichas quejas.

PALABRAS CLAVE: QUEJA ANTE UN TERCERO, EXPERIENCIA PREVIA, JUSTICIA INTERPERSONAL, FALLO.

\section{ABSTRACT}

Never before have so many services been bought, sold and consumed and consumers have had so many ways of expressing their dissatisfaction through a complaint. This paper studies hotel services complaints to a third party taking into account prior experiences of clients and the way they have been treated. The aim of this paper is to analyze how interpersonal justice, prior experience, gender and age could influence complaint intentions to a third party. Complaint instruments used by dissatisfied customers after a service failure are also analyzed, either newspapers, social networks, consumer associations or legal actions. Interesting results are presented for deducting consequences applicable to effective complaint management for the service sector plus recommendations to prevent such complaints.

KEYWORDS: THIRD PARTY COMPLAINT, PRIOR EXPERIENCE, INTERPERSONAL JUSTICE, FAILURE.

\section{INTRODUCCIÓN}

Según la información sobre consultas y reclamaciones por sectores económicos del Instituto Nacional de Consumo de España, para el año 2013 se registraron 1.249.747 quejas y reclamaciones 
(INC, 2014). En el sector de los servicios, estas quejas tienen en España un potencial cercano a los 3.000 millones de euros anuales (Méndez, 2013).

Para el sector empresarial la importancia de la gestión de las quejas radica en su valor como parámetro de la satisfacción de sus clientes y como oportunidades de mejoras en la gestión de sus procesos (Moliner, Berenger, Gil, y Fuentes, 2008; Álvarez, Vila, Fraiz, y De la Cruz, 2013). Estas quejas ante fallos en servicios tradicionalmente han sido clasificadas en tres categorías: quejas públicas, privadas y ante un tercero (Moliner y Fuentes, 2011). Las quejas públicas se presentan cuando el cliente se queja directamente con el proveedor del servicio, las quejas privadas son las quejas que el consumidor insatisfecho realiza ante sus amigos y familiares, mientras que las quejas ante un tercero son todas aquellas reclamaciones presentadas ante un tercer ente, es decir, una persona u organización que ni es la compañía de la que quiere quejarse ni pertenece a su grupo de amigos y familiares.

El tema de las quejas ante un tercero ha sido un fenómeno poco estudiado debido a que representan un pequeño porcentaje de las quejas totales. Sin embargo, se les debe prestar gran atención puesto que podrían suponer una cantidad considerable de dinero para la empresa (gastos en demandas) y tener fuertes implicaciones negativas en la imagen de la empresa y en sus relaciones públicas (Baker, Meyer y Chebat, 2013), además de derivar en costes legales excesivos, intervención reguladora y problemas para la reputación de la compañía (Tipper, 1997).

Los consumidores que se quejan han sido clasificados en cuatro categorías, siendo los más "agresivos" o activos los que se quejan ante un tercero. McAlister y Erffmeyer (2003) comentan que los consumidores tendrán una tendencia alta a presentar una queja ante un tercero cuando: a. perciben que la solución inicial de la compañía no fue la adecuada,

b. tienen buen acceso al sistema legal y otras agencias formales,

c. creen que los demás mecanismos de presentación de quejas no han tenido éxito,

d. experimentan altos niveles de ansiedad por la situación que provocó la queja, y

e. han generado actitudes negativas hacia las prácticas de la empresa.

Esta "agresividad" de los consumidores que se quejan, puede ser aumentada o disminuida de acuerdo con la experiencia previa y con el trato recibido durante la prestación de un servicio. La experiencia incluye la calidad y el número de experiencias anteriores con el proveedor del servicio, y el trato es el nivel de atención con que se cubren las necesidades de los consumidores, lo cual es de suma importancia ya que la atención es uno de los cuatro factores clave de los clientes en la evaluación de la experiencia percibida durante, por ejemplo, la estancia en un hotel (Hosteltur, 2013).

La relevancia del estudio de esta temática se debe a que nunca antes los consumidores habían tenido acceso a una variedad tan significativa de servicios para poder elegir el que más les convenga, ni tantos medios para poder expresar sus opiniones sobre el servicio brindado (por ejemplo, www.tripadvisor.es, www.booking. com, www.agenttravel.es, www.expedia.es, etc.). Por tanto, el control de las quejas debe ser entendido como un aspecto clave en la calidad de la prestación de los servicios en general y de los hoteleros en particular.

El interés de este trabajo se encuentra sustentado en las siguientes motivaciones:

1) Es de importancia crítica para la gerencia de un hotel, entender y responder las quejas de 
los clientes para evitar tanto su pérdida como que realicen publicidad negativa (Heung y Lam, 2003).

2) La entrega del servicio en este sector requiere un alto grado de acción interpersonal, por lo que se espera que una parte importante de la valoración de la experiencia se base en el trato brindado, y esto hace que para la evaluación del servicio se tengan en cuenta aspectos intangibles, como por ejemplo la empatía (Yuksel, Kilinc y Yuksel, 2006).

3) El interés de realizar investigaciones en este sector, debido al carácter dinámico del comportamiento del consumidor y sobre todo de la relevancia de su satisfacción (Laguna y Palacios, 2009).

Así, el objetivo principal de este trabajo es analizar qué influencia puede ejercer la justicia interpersonal, la experiencia previa, el género y la edad sobre la intención de quejarse ante un tercero ante el fallo de un servicio hotelero. Además, se busca identificar cuáles son los instrumentos de queja que utiliza un cliente insatisfecho tras un fallo, si los periódicos, las redes sociales, las asociaciones de consumidores o acometer acciones legales, y la tendencia a utilizar cada uno de estos medios dependiendo de la edad y el género. Para ello, en el siguiente apartado se realiza una revisión de los conceptos de justicia interpersonal, experiencia previa, quejas ante terceros y género y edad. A continuación se presenta la evidencia empírica de las quejas ante terceros para un caso concreto en el sector hotelero y se exponen los resultados obtenidos con el estudio. Finalmente, se plantean una serie de recomendaciones empresariales en cuanto a la gestión efectiva de las quejas e incluso recomendaciones para evitar que se interpongan dichas quejas.

\section{MARCO CONCEPTUAL Y FORMULACIÓN DE HIPÓTESIS}

\section{JUSTICIA INTERPERSONAL}

La justicia interpersonal se refiere a la percepción de justicia de los consumidores sobre la manera en la cual son tratados durante el proceso de la recuperación del servicio, por ejemplo, un trato que destaque por su amabilidad, pedir disculpas o brindar una explicación (Tax, Brown y Chandrashekaran, 1998; McColl-Kennedy y Sparks, 2003; Ok, Back y Shanklin, 2005).

Hui, Au y Zhao, (2007) argumentan que un efecto de la justicia interpersonal es que el consumidor tiende a estar más satisfecho en el intercambio social cuando siente que el proveedor del servicio es atento y cortés. Igualmente se ha demostrado que la justicia interpersonal es necesaria para entender mejor cómo el cliente forma sus percepciones de justicia (Bies y Shapiro, 1987; Aquino, 1995). Por otra parte, Shapiro y NiemanGonder (2006) exponen que muchos consumidores citan deficiencias interaccionales (pobre interacción con el personal de la empresa, falta de disculpa, ausencia de una explicación razonable, respuestas impersonales) como la causa de su insatisfacción con el servicio de recuperación. En una línea similar, Czepiel (1990) afirma que las relaciones interpersonales son especialmente importantes dado el alto grado de interacción personal, la naturaleza intangible del servicio, la heterogeneidad de los servicios brindados, y el prominente rol que el consumidor juega en la elaboración del servicio. Aguilar y Fandos (2013) exponen que independientemente de la tecnología disponible, la compensación que se pueda ofrecer, o la rapidez con que se pueda atender, si el consumidor no siente que se le ha tratado de una forma respetuosa y amable entonces es sumamente difícil lograr su satisfacción. 


\section{EXPERIENCIA PREVIA}

La experiencia previa del consumidor con el servicio ha sido un factor determinante para establecer un vínculo entre consumidores y proveedores. Kim, Choi y Han (2009) afirman que la experiencia previa con un servicio cambia las percepciones y predice un comportamiento tendente a favorecer al proveedor. Este vínculo se explica mediante la teoría de involucramiento del consumidor (Laurent y Kapferer, 1985), la cual justifica la repetición o revisita debido a una familiaridad preexistente, producto de experiencias anteriores directas, logrando que el consumidor se sienta comprometido y perciba un menor riesgo ante compras futuras. De manera similar, la teoría de referencias cognitivas (Folger, 1987) argumenta que una persona evalúa niveles de trato basándose en eventos pasados.

Según Namkung, Jang y Choi (2011) la experiencia previa es un factor que debe ser considerado cuando se estudian las tendencias de comportamiento del consumidor tras un fallo en el servicio. Prueba de lo anterior es que el fallo en el servicio tiene consecuencias más negativas en clientes nuevos que en clientes habituales (Ruyter y Wetzels, 2000; San Martín, Collado y Rodríguez, 2008). En una línea similar, Bolton, Grewal y Levy (2007) afirman que los consumidores tienen menos tendencia a estar insatisfechos tras un fallo cuando han tenido experiencias previas excelentes con la organización.

\section{QUEJAS ANTE UN TERCERO}

Cuando un consumidor no está conforme con el rendimiento del servicio brindado, dispone de varias opciones para manifestar su insatisfacción (Singh, 1988). Según este autor, el cliente puede: abandonar la relación con el proveedor, irse a la competencia, reclamar directamente al proveedor, quejarse ante amigos y familiares o elevar una reclamación ante un tercero.
Las quejas ante un tercero son todas aquellas realizadas ante cualquier ente que no sea la propia compañía, ni amigos, ni familiares (Vásquez, 2011). Difundir las quejas a través de un tercero puede ser un comportamiento comprensible cuando los consumidores están insatisfechos con las respuestas de la compañía sobre el fallo del servicio (Reiboldt, 2003). Cuando el consumidor se queja ante un tercero puede deberse a: un ineficiente sistema de administración de quejas, falta de respuesta eficaz y oportuna e inclusive, un juicio poco ético por parte del proveedor del servicio (McAlister y Erffmeyer, 2003). Las quejas que se tramitan ante un tercero vienen de consumidores que no encontraron una solución con el proveedor o percibieron tanta gravedad en el problema, que consideraron que su insatisfacción no era un caso aislado y que podría involucrar a otros consumidores (Hogarth, English y Sharma, 2001).

En cuanto a la percepción de un trato injusto, los consumidores que se han quejado y perciben una ausencia de justicia en el trato con el proveedor tienen una tendencia más alta a quejarse ante un tercero (McAlister y Erffmeyer, 2003). Cuando un consumidor está insatisfecho, denunciar ante un tercero dependerá de su percepción de justicia (Blodgett, Hill y Tax, 1997). Schoefer y Diamantopoulos (2008) argumentan que el hecho de quejarse ante un tercero es el resultado directo de un proceso de juicio cognitivo (percepción de justicia, por ejemplo): si no se ha experimentado esta percepción de justicia en el encuentro de recuperación del servicio, entonces el consumidor tomará esta decisión para que la justicia sea restaurada.

Con respecto a la experiencia previa, según Lerman (2006) las expectativas y experiencias previas del consumidor constituyen uno de los antecedentes para tener una tendencia más alta a plantear una queja ante un tercero. De manera similar, Moliner, Fuentes, Gil y Berenguer (2010) argumentan que la intención de quejarse ante 
un tercero será mayor o menor dependiendo de la experiencia previa con la que cuente el consumidor. Basándonos en los argumentos expuestos se proponen las siguientes hipótesis:

H1) La intención de quejarse ante un tercero será menor si el sistema de recuperación del servicio cuenta con elementos de justicia interpersonal.

H2) La intención de quejarse ante un tercero será menor si el consumidor cuenta con experiencia previa.

La razón de que muchas veces el consumidor elija exponer su queja ante un tercero es que el proveedor ha fallado a la hora de disponer de los medios adecuados para que los consumidores le den retroalimentación sobre el servicio brindado; o bien, que el consumidor percibe que el grado de su insatisfacción es tan alto que debe procurar exponer el caso de la manera que afecte más al proveedor del servicio y que la queja pueda ser vista por un mayor número de personas. Existe un consenso en proponer que entre las modalidades más utilizadas de quejas ante un tercero se encuentran: quejas en periódicos, quejas en redes sociales, quejas ante asociaciones de consumidores y tomar acciones legales (Figura 1).

\section{QUEJAS EN PERIÓDICOS}

Durante años, quejarse por medio de los periódicos ha sido una herramienta muy utilizada por los consumidores, debido principalmente a su utilidad y fácil acceso para los usuarios. Liu y McClure (2001) argumentan que las quejas ante un periódico a pesar de ser públicas se hacen ante un ente externo, y por eso se considera que exponer la queja en una carta a un periódico es una queja ante un tercero. Los dos tipos de quejas principales que se presentan en un periódico son 1) ante algún proveedor de un servicio privado, o 2) ante una autoridad pública.

En cuanto a quejas sobre un proveedor de servicio privado, Hannigan (1977) muestra un trabajo en el cual se estudia qué tipo de consumidores exponen su queja ante el periódico y qué solución obtuvieron en este medio. Esta misma acción por parte de los consumidores ha sido adaptada para diversos estudios (Kitapci, y Dortrol, 2009; Moliner et al., 2010; Baker et al., 2013).

Con respecto a quejas ante autoridades públicas, Henry y Ho (2010) muestran en un estudio la utilidad de quejarse en los periódicos contra autoridades públicas, ya que al ser publicadas y leídas animaban a otros clientes a quejarse por este mismo medio.

FIGURA 1

\section{ANTECEDENTES QUEJAS ANTE UN TERCERO}

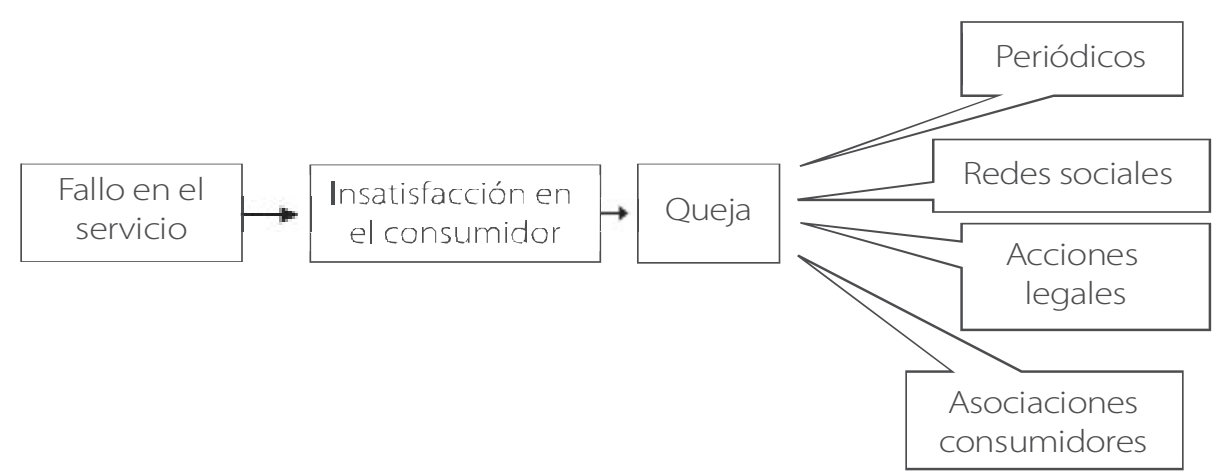

Fuente: Elaboración propia. 


\section{QUEJAS EN REDES SOCIALES}

Como consecuencia del fácil y masivo acceso a Internet que tienen hoy los consumidores (Europa Press, 2011) y de la gran popularidad de las redes sociales (Sanpedro, 2012), la posibilidad de quejarse ante un tercero está al alcance de cualquier consumidor insatisfecho. Cualquier persona puede transmitir un mensaje a miles (ó millones) de personas mediante Facebook, Twitter, Linkedin con la ventaja de poder realizar esta comunicación en "tiempo real" y que otras personas tengan la posibilidad de reenviar el mensaje, obligando a las empresas a que ofrezcan productos personalizados y un inmediato feedback (Del Águila-Obra et al., 2012).

Sin embargo, el setenta por ciento de las compañías ignoran las quejas de los clientes en Twitter (Baer, 2011), por lo que demuestran una falta de preparación o interés en esta área. Un ejemplo concreto lo exponen Van Noort y Willemsen (2011): Un famoso comediante holandés publicó mediante su cuenta experiencias negativas con el servicio de un cliente de una compañía telefónica. Esta se disculpó vía Twitter, lo que el comediante consideró una respuesta inadecuada. Como contestación, él empleó las redes sociales para ventilar sus sentimientos negativos con respecto a la compañía e invitaba a sus seguidores a hacer lo mismo, creando un círculo vicioso de Word of Mouth (recomendaciones personales) negativo.

De manera similar, mediante Facebook los consumidores pueden comparar sus opiniones en términos de calidad y precio sobre productos y servicios con las de otros compradores, y determinar si su compra fue justa (Wu, 2013), o también crear páginas del tipo "Odio los call centers" con lo que dejan bastante clara su insatisfacción al tratar con estos servicios (Honeycutt et al., 2012).

\section{QUEJAS ANTE ASOCIACIONES DE CONSUMIDORES}

Las asociaciones de protección al consumidor son organizaciones sumamente útiles en la atención y tramitación de quejas (Heung y Lam, 2003), dando un especial énfasis en los informes de los problemas con los que se encuentran los consumidores en sus relaciones con proveedores de productos y servicios.

Por otra parte, en muchas ocasiones las compañías retienen o posponen la solución de las reclamaciones para desalentar la presentación de quejas. Sin embargo, desde que estas demandas se solicitan ante agencias de protección al consumidor, las empresas muchas veces se ven obligadas a dar una solución, incluso cuando no están de acuerdo con la apelación interpuesta (Ryngelblum, Vianna y Rimoli, 2013).

Más aún, en el contexto de la defensa de las reclamaciones de los clientes, las agencias de protección del consumidor enfatizan el valor del comportamiento de queja, al mostrar cómo al proveer información sobre productos defectuosos o servicios de mala calidad, se protege a otros consumidores de posibles daños (Chelminski y Coulter, 2011).

\section{ACCIONES LEGALES}

Los consumidores tienen la posibilidad de tomar acciones legales contra una compañía en general, pero son aquellos que han acumulado un alto grado de insatisfacción, generándoles actitudes negativas hacia la misma, y que además conocen ampliamente sus derechos en el mercado, los que más habitualmente lo hacen (Tipper, 1997).

Los consumidores podrían mostrar este nivel de insatisfacción debido al fallo en el servicio con comportamientos sumamente serios y extremos que conllevan el establecimiento de una demanda (Baker et al., 2013), ya que el consumidor ha decidido que esta es la mejor manera de obtener 
una respuesta del proveedor para lograr alcanzar algún tipo de compensación.

Cuando se ha realizado una compra significativa, el consumidor tendrá una probabilidad más alta de interponer, no solamente una queja con el proveedor, sino una demanda legal si fuera necesario. Si la compra ha sido de una trascendencia muy destacada (esfuerzo grande en cuanto a dinero y tiempo o significado emocional) los clientes podrían pasar por alto al proveedor e ir directamente hacia un tercero, buscando restaurar su satisfacción y generarle al proveedor el pago de multas y honorarios legales (Ferguson y Johnston, 2011).

\section{GÉNERO Y EDAD}

El género y la edad han despertado un especial interés en el estudio del marketing desde que se ha comprobado que tienen un importante impacto en áreas como el procesamiento de información, la toma de decisiones y el proceso de compra (Phillips y Sternthal, 1977; Tongren, 1988; lacobucci y Ostrom, 1993; Simcock Sudburyy y Wright, 2006; Han, Hsu y Lee, 2009; Hernández, Jiménez y Martín, 2011). Desde la perspectiva del proveedor del servicio, tanto el género como la edad son empleados con frecuencia como una variable de segmentación, debido a que son fácilmente identificables y accesibles (Homburg y Giering, 2001; Yelkur y Chakrabarty, 2006).

En cuanto al género, la Teoría del Rol Social (Eagly, 1987) asume que los individuos tienden a aceptar de forma pasiva roles que se les han impuesto, por lo que hombres y mujeres socializan y se comportan de una manera diferente en nuestra sociedad (Han et al., 2009). Como consecuencia de estas diferencias, las expectativas de hombres y mujeres son distintas, y se han ido transmitiendo a las nuevas generaciones, influyendo en el comportamiento social de cada género (Eagly, Wood y Diekman, 2000).
Con respecto a la edad, Levinson (1986) argumentó que de acuerdo a las Teorías de la Edad Social, los cambios en las relaciones y los roles sociales experimentados por la gente van cambiando a lo largo de sus vidas (Moschis, 1994). Estos cambios en el comportamiento social son producidos por su decisión de adaptarse al contexto y la sociedad en la cual vive (Chadha y Willigen, 1999).

Además, distintos estudios han argumentado que las diferencias de género y edad podrían influir en el comportamiento pos-queja y en la satisfacción con el servicio de recuperación (Han, Keng y Richmond, 1995; McColl-Kennedy, Daus y Sparks, 2003; Kalamas, Laroche y Makdessian, 2008; Mattila, 2010). Con base en los argumentos expuestos anteriormente, proponemos las siguientes hipótesis:

H3) La intención de quejarse ante un tercero será significativamente diferente en función del género del consumidor.

H4) La intención de quejarse ante un tercero será significativamente diferente en función de la edad del consumidor.

La Figura 2 presenta el modelo conceptual propuesto. En él, se plantea la existencia de una relación entre la justicia interpersonal percibida por el consumidor y su nivel de experiencia previa con la intención de quejarse ante un tercero y cómo las diferencias en el género y la edad del consumidor afectan esta relación.

\section{METODOLOGÍA}

Para desarrollar el estudio empírico se tomó como referencia el sector hotelero en España, ya que este es un sector donde los fallos y el comportamiento post-compra son frecuentes (Ambrose, Hess y Ganesan, 2007). Además, pertenece a un área económica que es de vital importancia para este país (Alberca y Parte, 2013), representando aproximadamente el $12 \%$ del PIB 


\section{MODELO CONCEPTUAL PROPUESTO}

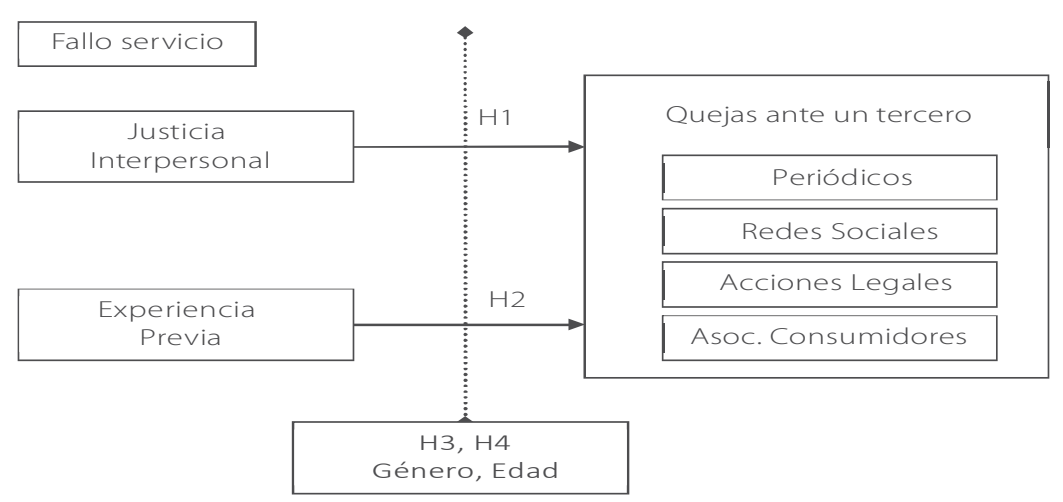

Fuente: Elaboración propia.

y del empleo (Parte y Such, 2011), siendo la hostelería la rama que más peso tiene en el total de actividades turísticas (IET, 2016).

Para identificar la tendencia a quejarse ante un tercero, se plantea un diseño experimental de cuatro escenarios sobre un grupo de personas que hubieran estado hospedados en un hotel al menos una vez en los últimos seis meses. Esta condición fue propuesta para que los individuos se identificaran en mayor medida con el escenario a analizar. Los sujetos fueron asignados aleatoriamente en un escenario de cuatro posibles, dentro de un diseño entre-sujetos de 2 (trato justo vs trato no justo) $\times 2$ (experiencia previa vs no experiencia previa) condiciones.

En la tabla 1, se presenta el perfil demográfico de la muestra y su distribución en los cuatro escenarios propuestos. La selección de la muestra se llevó a cabo mediante un muestreo aleatorio de estudiantes de pregrado, grado y posgrado de la Universidad de Zaragoza a los cuales se les aplicó la encuesta. La muestra es de 352 personas, de los cuales 201 fueron hombres y 151 mujeres. En cuanto a la edad, 112 son menores de 23 años, 138 tienen entre 23 y 30 años, mientras 102 son mayores de 30 años. Las edades abarcan valores desde los 18 años hasta los 52 (estudiantes posgrado).
En cuanto al diseño del experimento, se planteó una situación en la que el consumidor al llegar al hotel se encontraba con que el recepcionista le decía que su reserva no había sido registrada. Para reclamar dicho fallo en la entrega del servicio, el encuestado se situaba en cuatro posibles escenarios respecto a la experiencia previa y al trato del que fue objeto. Los escenarios han sido manipulados de la siguiente forma:

1) El trato brindado al consumidor se manipuló en dos niveles:

Nivel A: El gerente del hotel llega pronto a atender al cliente y lo trata de una manera muy amable resolviéndole de inmediato la situación

Nivel B: El gerente del hotel hace esperar al cliente y cuando llega lo trata de una manera despectiva y no queda claro que le vaya a resolver el problema

2) La experiencia previa se manipuló en dos niveles:

Nivel A: Es la cuarta vez que el cliente va al hotel y las veces anteriores la estancia fue excelente

Nivel B: Es la primera vez que el cliente va al hotel

Una vez planteados los escenarios, se les pidió a los individuos que valorasen en una escala Likert 
TABLA 1

\section{PERFIL DE LA MUESTRA}

\begin{tabular}{|c|c|c|c|}
\hline \multirow{3}{*}{ Muestra } & \multicolumn{2}{|c|}{352} & \multicolumn{2}{|c|}{ Escenarios } & 352 \\
\hline \multirow{3}{*}{ Género } & Hombres: $57 \%$ & No experiencia - No Justicia & 100 \\
\cline { 2 - 4 } & Mujeres: $43 \%$ & No experiencia - Sí Justicia & 81 \\
\hline \multirow{3}{*}{ Edad } & $<=22: 31,8 \%$ & Sí experiencia - No Justicia & 84 \\
\cline { 2 - 4 } & $>22 y<=30: 39,2 \%$ & Sí experiencia - Sí Justicia & 87 \\
\cline { 2 - 4 } & $>30: 29 \%$ & & \\
\hline
\end{tabular}

Fuente: Elaboración propia.

del 1 al 7 (donde indican (1) si está "Totalmente en desacuerdo" o (7) si está "Totalmente de acuerdo"), la tendencia a presentar una queja ante un tercero de acuerdo a los siguientes ítems (ver tabla 2). La fiabilidad de la escala se analizó a través del coeficiente de Cronbach, considerando ur valor mínimo de 0,7 (Nunally, 1978). La escala ha superado este mínimo $(0,805)$.

\section{TABLA 2}

\section{ESCALA DE MEDIDA}

\begin{tabular}{l} 
Quejas ante un tercero (Liu y McClure, 2001) \\
\hline 1) Escribiría una carta al periódico contando la experiencia \\
\hline 2) Contaría en las redes sociales la experiencia con el hotel * \\
\hline 3) Haría llegar el problema a una asociación de consumidores \\
\hline 4) Emprendería alguna acción legal contra el hotel \\
\hline
\end{tabular}

* = ítem de elaboración propia.

Por otra parte, se aplicó una prueba KolmogorovSmirnov para averiguar si la escala tiene una distribución normal (ver tabla 3). Dado que el valor de la probabilidad (Sig) asociada a la distribución empírica de cada uno de los escenarios de análisis es mayor que 0,05, se tiene evidencia estadística para afirmar que la escala observada proviene de una población con distribución normal.

Para responder a las cuestiones de investigación planteadas se ha calculado la media de los ítems de la variable quejas ante un tercero (Tabla 4), tras comprobar que dicha escala goza de la necesaria fiabilidad. Posteriormente, mediante la aplicación de un ANOVA se ha comprobado mediante la prueba $F$ la existencia de diferencias significativas en las medias de las muestras de los distintos escenarios (Tabla 4).

\section{RESULTADOS}

En la tabla 5 se observan los resultados de la comparación de los casos de escenarios sin justicia interpersonal $(\mathrm{N}=184)$ contra los casos de escenarios que sí la contemplan ( $N=168$ ). La hipótesis 1 (La intención de quejarse ante un tercero será menor si el sistema de recuperación del servicio cuenta con elementos de justicia interpersonal) se confirma. Los resultados obtenidos ponen de manifiesto que existen diferencias significativas en la intención de quejarse ante un tercero, en un ambiente en el que los consumidores perciben que son tratados con amabilidad y respeto contra un ambiente en el que esta situación no se presenta. Estos hallazgos están en la misma línea de otros trabajos (por ejemplo, Hui et al., 2007; Namkung y Jang, 2009) que constatan que a mayor justicia interpersonal, mejor el comportamiento del consumidor hacia el proveedor del servicio. 
TABLA 3

PRUEBA DE NORMALIDAD

\begin{tabular}{|c|c|c|c|}
\cline { 2 - 4 } \multicolumn{1}{c|}{} & \multicolumn{3}{c|}{ Kolmogorov-Smirnov } \\
\hline Escenario & Estadístico & gl & Sig. \\
\hline No experiencia - No Justicia & 0,099 & 100 & 0,077 \\
\hline No experiencia - Sí Justicia & 0,212 & 81 & 0,086 \\
\hline Sí experiencia - No Justicia & 0,11 & 84 & 0,094 \\
\hline Sí experiencia - Sí Justicia & 0,12 & 87 & 0,068 \\
\hline
\end{tabular}

Fuente: Elaboración propia.

TABLA 4

TENDENCIA QUEJA ANTE UN TERCERO POR ESCENARIOS

\begin{tabular}{|c|c|c|c|c|} 
Escenario & N & Media & $F$ & P \\
No experiencia - No Justicia & 100 & 3,67 & 8,366 & 0,000 \\
\hline No experiencia - Sí Justicia & 81 & 2,75 & & \\
\cline { 1 - 1 } Sí experiencia - No Justicia & 84 & 3,42 & \\
\cline { 1 - 2 } Sí experiencia - Sí Justicia & 87 & 2,59 & \\
\hline
\end{tabular}

Fuente: Elaboración propia.

TABLA 5

TENDENCIA A QUEJARSE SEGÚN LA JUSTICIA INTERPERSONAL

\begin{tabular}{|c|c|c|c|c|}
\hline Escenarios & $N$ & Media & F & P \\
\cline { 1 - 2 } Sin Justicia Interpersonal & 184 & 3,56 & 23,61 & 0,000 \\
\hline Con Justicia Interpersonal & 168 & 2,66 & & \\
\cline { 1 - 2 } & & &
\end{tabular}

Fuente: Elaboración propia.

Los resultados de la comparación entre los escenarios sin experiencia previa $(N=181)$ y aquellos que sí la contemplan $(N=171)$ se muestran en la tabla 6 . No se tiene suficiente evidencia estadística para probar la hipótesis 2, la cual estable que la intención de quejarse ante un tercero será menor si el consumidor cuenta con experiencia previa.
TABLA 6

\section{TENDENCIA A QUEJARSE SEGÚN LA EXPERIENCIA PREVIA}

\begin{tabular}{|c|c|c|c|c|}
\hline \multicolumn{1}{|c|}{ Escenarios } & N & Media & $F$ & F \\
\cline { 1 - 3 } Sin experiencia previa & 181 & 3,26 & 1,975 & 0,161 \\
\hline Con experiencia previa & 171 & 2,99 & & \\
\cline { 1 - 2 } & &
\end{tabular}

Fuente: Elaboración propia.

De acuerdo a estos datos, se deduce que, sin importar las experiencias anteriores con el proveedor del servicio, el consumidor percibe que el daño está hecho, y por ende, las diferencias no son significativas. Este resultado podría justificarse mediante la Teoría de la Justicia (Rawls, 1971) la cual establece que un consumidor evalúa la recuperación del servicio como justa o injusta (Dewitt, Nguyen y Marshall, 2008), es decir, en el preciso momento de esta evaluación podría pesar más el fallo en sí, que las experiencias anteriores. 
En la tabla 7 se muestran los resultados con respecto a la intención de quejarse ante un tercero por género y por edad. En cuanto al género, las mujeres tienden en promedio a reclamar más a un ente externo que los hombres, pero la diferencia no llega a ser significativa, por lo que no existe evidencia estadística a favor de la hipótesis la hipótesis 3 (La intención de quejarse ante un tercero será significativamente diferente en función del género del consumidor).

TABLA 7

INTENCIÓN DE QUEJARSE ANTE UN TERCERO (POR GÉNERO)

\begin{tabular}{|c|c|c|c|c|}
\hline Escenarios & \multicolumn{1}{|c}{$N$} & Media & $F$ & $P$ \\
\cline { 1 - 2 } Mujer & 151 & 3,2 & 0,389 & 0,533 \\
\hline Hombre & 201 & 3,08 & \multicolumn{2}{|c|}{} \\
\cline { 1 - 2 } & &
\end{tabular}

Fuente: Elaboración propia.

Por otra parte, la hipótesis 4 sí se confirma (tabla 8). Así, se dispone de evidencia estadística para afirmar que la intención de quejarse ante un tercero es diferente en función de la edad del consumidor. Se observa que a mayor edad, mayor intención de quejarse ante un tercero. Estos hallazgos están de acuerdo con la Teoría de Estratificación por Edad (Riley, Johnson y Foner, 1972), la cual argumenta que a mayor edad la persona acumula más experiencia y tiene acceso a más recursos (contactos, información, recursos económicos e influencia social).

TABLA 8

INTENCIÓN DE QUEJARSE ANTE UN TERCERO (POR EDAD)

\begin{tabular}{|c|c|c|c|c|}
\hline Escenarios & N & Media & $F$ & $p$ \\
\hline$<=22$ & 112 & 2,71 & 5,65 & 0,004 \\
\hline$>22 y<=30$ & 138 & 3,3 & & \\
\hline$>30$ & 102 & 3,43 & & \\
\hline
\end{tabular}

Fuente: Elaboración propia.
Asimismo, para profundizar más en el tema, se busca identificar cuál es la tendencia a utilizar cada una de las opciones de queja ante un tercero (periódicos, redes sociales, acciones legales, asociación de consumidores) dependiendo del escenario (trato, experiencia), de la edad y del género.

Para mostrar el resultado de los datos sobre cuál es la tendencia a quejarse ante un tercero y qué medios utilizan los consumidores para llevarlo a cabo se presenta la figura 3, el cual muestra la tendencia a utilizar los distintos medios para interponer una queja ante un tercero según la calificación promedio en la escala de quejas. Los consumidores que eligen las redes sociales para quejarse muestran un promedio más alto en la escala de quejas ante un tercero, lo cual resulta deducible ante el gran aumento del acceso a Internet y la repercusión de las redes sociales, aunado a la facilidad para cualquier consumidor de escribir desde su teléfono celular, computadora o tablet. Todo ello, con la certeza de que su descontento podría ser observado por miles de personas, muchas de las cuales se podrían involucrar en el mismo comportamiento, ya sea por solidaridad o por empatía, ya que probablemente también han sido "víctimas" de ese proveedor.

El segundo lugar lo ocupan las asociaciones de consumidores. Estas son entidades privadas sin ánimo de lucro, cuya finalidad es la protección y defensa de los intereses de los consumidores, tanto en sentido general como en relación con productos o servicios determinados.

En tercer lugar, con una media de 2,48 , se encuentran las quejas en los periódicos. Los consumidores aprecian que este medio continúa siendo muy popular, ya que la información de las reclamaciones llega a un público masivo que lee el periódico. Finalmente, los consumidores presentarían una acción legal en menor medida (una media de 2,4). Como era de esperar, ocupan el último lugar debido a que, para un consumidor, 
establecer una demanda legal representa un mayor costo, tanto económico como de tiempo, en comparación con las posibilidades planteadas, para presentar una queja.

La segunda cuestión a investigar trata de averiguar la tendencia a quejarse ante un tercero dependiendo tanto del trato brindado por el proveedor del servicio (justicia interpersonal) como de la experiencia previa con el mismo. La tabla 9 muestra la tendencia a interponer una queja ante un tercero según los 4 escenarios propuestos. De estos, el mayor promedio a quejarse ante un tercero se muestra en el escenario en el cual al consumidor se le trata mal, además de no tener experiencia previa con el proveedor (en una escala del 1 al 7 la media de queja ante un tercero es de 3,67). El promedio más bajo se presenta si el consumidor tiene experiencia previa con el proveedor del servicio y se le ha tratado bien (media de 2,59).
En la figura 4, se muestra la tendencia a quejarse de acuerdo a los escenarios propuestos en los que se relacionan la experiencia con la justicia. En un escenario en el cual el cliente ha sido tratado con justicia, además de tener experiencia previa con el proveedor del servicio, hay claramente menos tendencia a quejarse ante un tercero (periódico media de 1,94; redes sociales: 3,59; asociación consumidores: 2,72; acción legal: 2,09). Por otra parte, en la prestación de un servicio donde el cliente no tiene experiencia previa con el proveedor ni se recibe un buen trato, la tendencia a quejarse ante un tercero es más alta (periódico media de 2,86; redes sociales: 4,85; asociación consumidores: 4,08; acción legal: 2,88).

Para continuar, consideramos muy interesante observar si determinadas características de los consumidores, como la edad o el género, pueden resultar decisivas a la hora de utilizar un medio u otro de queja.

En primer lugar, se analizó la tendencia a quejarse ante un tercero por edades, cuyos resultados se

\section{FIGURA 3}

\section{INSTRUMENTOS USADOS EN QUEJA ANTE UN TERCERO}

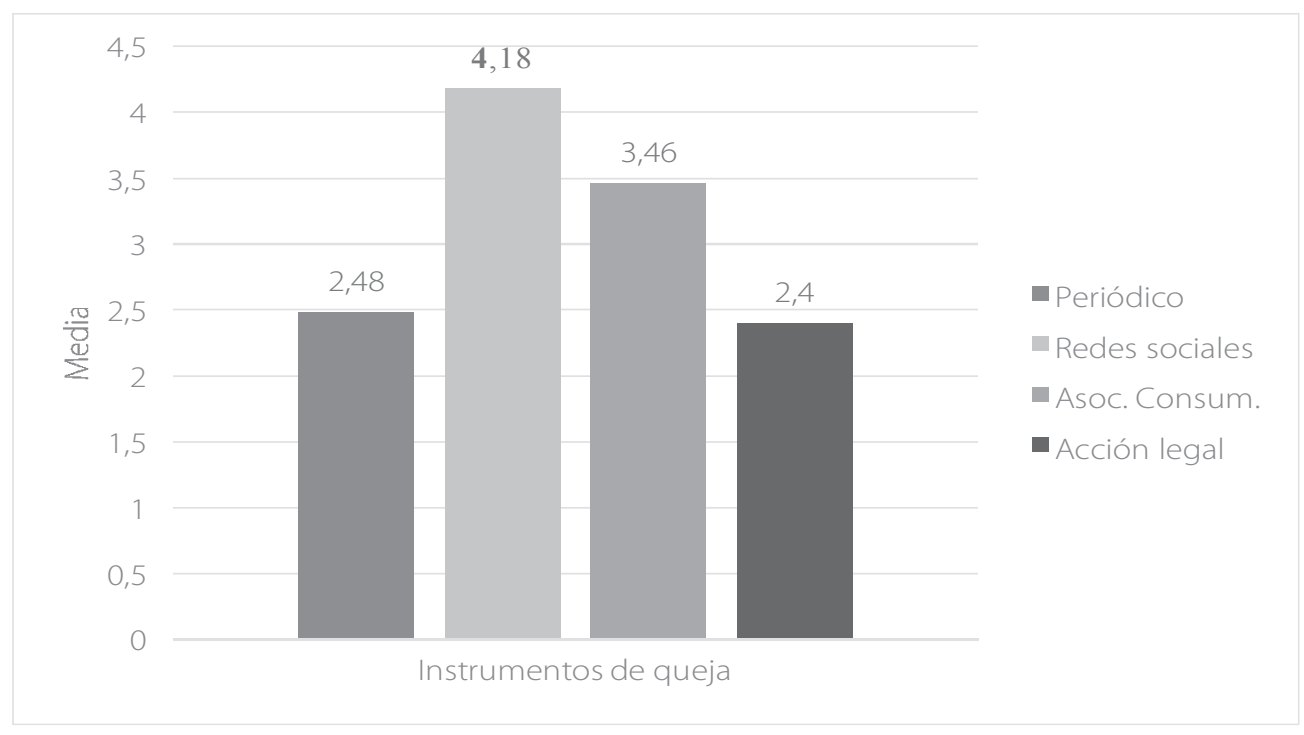

Fuente: Elaboración propia. 
TABLA 9

\section{TENDENCIA QUEJA ANTE UN TERCERO POR ESCENARIOS}

\begin{tabular}{|c|c|c|c|c|}
\hline Escenario & N & Media & F & P \\
\cline { 1 - 2 } No experiencia - No Justicia & 100 & 3,67 & 8,366 & 0,000 \\
\hline No experiencia - Sí Justicia & 81 & 2,75 & & \\
\cline { 1 - 2 } Sí experiencia - No Justicia & 84 & 3,42 & & \\
\cline { 1 - 2 } Sí experiencia - Sí Justicia & 87 & 2,59 & & \\
\cline { 1 - 2 } & & & &
\end{tabular}

Fuente: Elaboración propia.

FIGURA 4

TENDENCIA A QUEJARSE ANTE UN TERCERO DE ACUERDO

A LA EXPERIENCIA Y EL TRATO

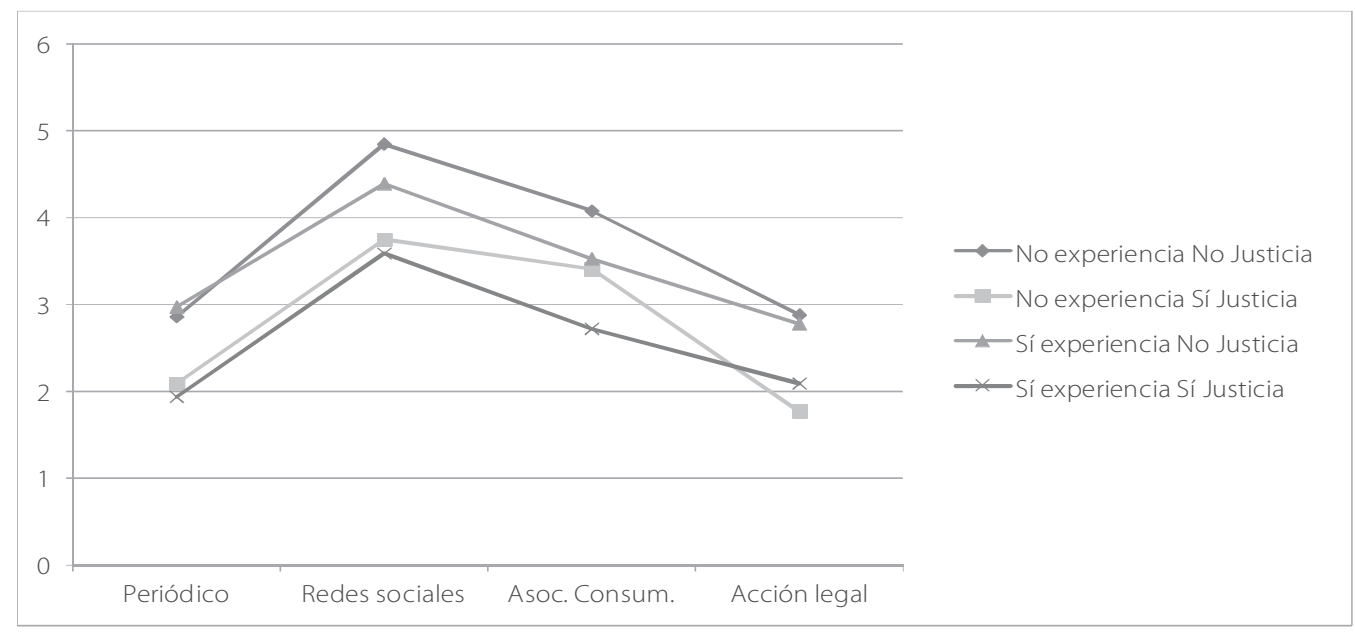

Fuente: Elaboración propia.

muestran en la figura 5. Las personas con edades comprendidas entre los 22 y los 30 años (138 de la muestra de 352) han manifestado tener mayor tendencia a quejarse mediante las redes sociales y a presentar reclamaciones ante alguna agencia de protección de los derechos de los consumidores. Mientras tanto, los mayores de 30 años (102 de la muestra de 352) muestran mayor disposición a interponer una queja mediante un periódico o tomar alguna acción legal contra la empresa. Esto se justifica al contemplar que una persona con mayor edad podría tener más acceso a información, contactos y recursos para involucrarse en un proceso de demanda contra una empresa.

Finalmente, se pretende averiguar cuál es la opción utilizada para quejarse ante un tercero dependiendo del género. Estos resultados se muestran en el gráfico 4. En general, los hombres y las mujeres presentan valores muy similares, teniendo los primeros una ligera tendencia a quejarse más ante asociaciones de consumidores (media de 3,52; mujeres: 3,38 ) y de tomar acciones legales (media de 2,42; mujeres: 2,39); mientras que ellas tienen una tendencia más alta a quejarse en redes 
sociales (media de 4,24; hombres: 4,13). Además, sobresale el resultado obtenido para las quejas ante periódicos, donde se ha obtenido una mayor diferencia (mujeres: 2,78; hombres: 2,26).

\section{CONCLUSIONES E IMPLICACIONES PARA LA GESTIÓN}

En el presente estudio se ha demostrado la influencia que tiene la justicia interpersonal y la experiencia previa en el comportamiento de queja del consumidor. Se observa que un buen trato y un proveedor conocido disminuyen la tendencia a quejarse ante un tercero, pues en un "escenario ideal" en el cual hay justicia interpersonal la tendencia a quejarse ante un tercero es la más baja de los 4 escenarios planteados (2,59 sobre 7). Por lo tanto, elementos como la cortesía, empatía, amabilidad, buena comunicación, son factores favorables, y en caso de cometer un fallo, las disculpas y explicaciones sobre el origen del inconveniente ocurrido harán posible mantener la lealtad y satisfacción del consumidor, disminuyendo con esto la posibilidad de que presente una queja ante un tercero. Además, tal y como lo señalan González, Alén y Rodríguez (2011) la existencia de procedimientos para recoger quejas de los clientes es una herramienta vital para la certificación de la calidad de la empresa.

Con respecto a la edad, los consumidores mayores de 30 años son quienes tienen una tendencia más alta a quejarse ante un tercero. Las razones de esta conducta pueden guardar un vínculo con las oportunidades de información, contactos, y recursos económicos que usualmente aumentan con la edad.

En cuanto al género, si bien las diferencias no son significativas, los resultados están de acuerdo con el argumento de que en general las mujeres se quejan más (Kalamas, Laroche y Makdessian, 2008).

Por otra parte, la insatisfacción de los clientes puede redundar en consecuencias dañinas para las empresas. Por lo general, cuando un

FIGURA 5

TENDENCIA A QUEJARSE ANTE UN TERCERO POR EDADES

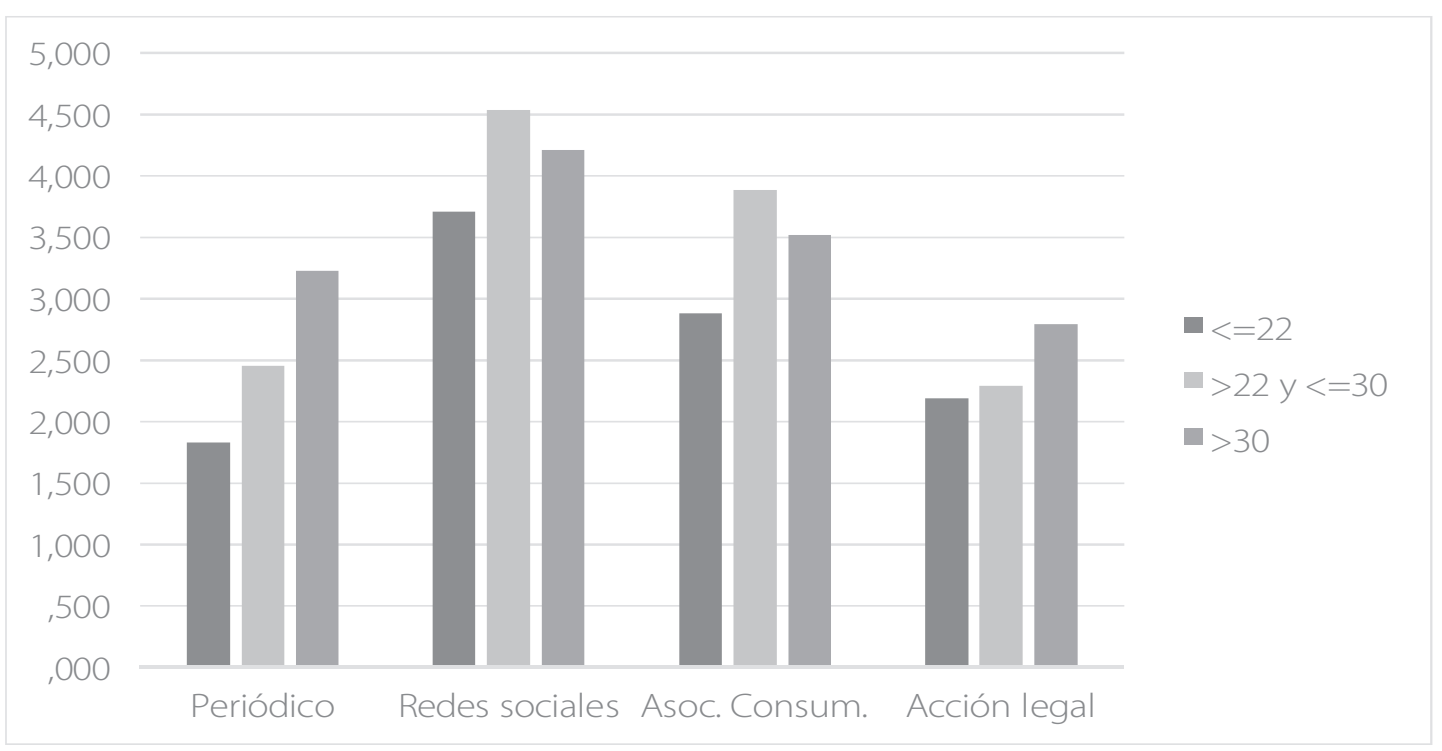

Fuente: Elaboración propia. 


\section{TENDENCIA A QUEJARSE ANTE UN TERCERO DE ACUERDO AL GÉNERO}

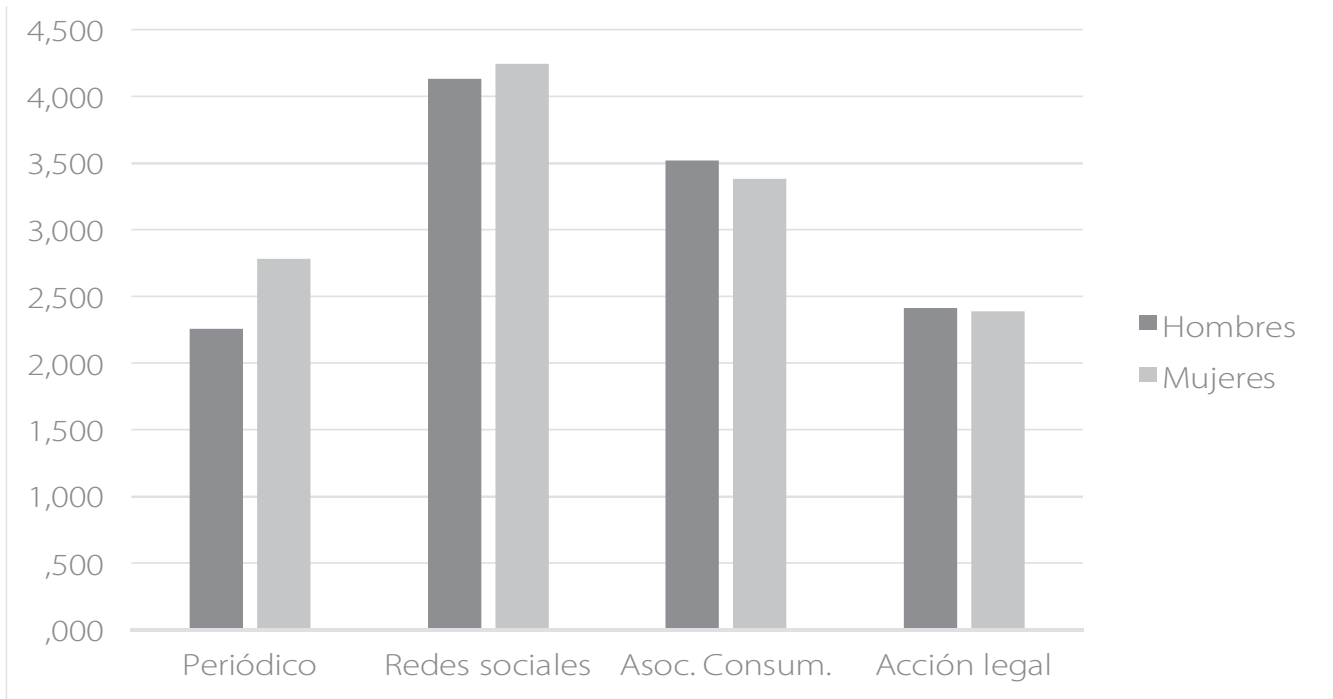

Fuente: Elaboración propia.

consumidor se queja ante un tercero su reclamación siempre tendrá un mayor alcance que si se quejara ante el propio proveedor del servicio o ante un grupo reducido de amigos y/o familiares. Algunas de estas consecuencias que las empresas de servicios tienen que considerar para una gestión efectiva serían:

- Daño en la reputación de la empresa: al salir a la luz pública esta reclamación se podría crear la percepción de que esta empresa no se preocupa por sus clientes.

- Pérdida de clientes: los clientes actuales de la compañía pueden pensar que esa misma situación que le pasó les podría pasar a ellos, por lo que se plantean cambiar de proveedor hacia uno del que no hayan escuchado alguna queja.

- Pérdida de clientes potenciales: consumidores potenciales de la compañía, se pue- den sentir persuadidos para no adquirir ningún servicio o producto y así evitar la misma situación de la persona que ha presentado esta queja.

- Pérdida de recursos: en el caso de tener que atender una demanda legal se deben incurrir en costes económicos y de personal (abogados, notarios, etc.).

El proveedor del servicio debe ser consciente de que en cualquier momento se puede presentar un fallo, por lo tanto, en lugar de pensar en una operación "a prueba de fallos" debe buscar el escenario ideal: que el cliente le presente directamente sus reclamaciones o recomendaciones. Para evitar que un consumidor interponga una queja ante un tercero, la empresa debería incluir en el protocolo del diseño de la prestación de sus servicios:

- Proveer al consumidor herramientas de fácil acceso para que pueda interponer una 
queja o recomendación de mejora directamente en la empresa. Tal y como señalan Vázquez-Casielles, del Río-Lanza y SuárezÁlvarez (2009), manejar las quejas de los consumidores es una función indispensable en la calidad del servicio. Por ello, se deben proveer medios para que el cliente se queje directamente con el proveedor: hojas de reclamación, página en Facebook, cuenta en Twitter, correo electrónico, teléfono, atención personal en alguna oficina, etc.

- Tener un mecanismo que responda de manera ágil y rápida a cada reclamación, logrando que el consumidor no solo perciba que se le da importancia a sus quejas sino que también recibe una respuesta.

- Ofrecer a los clientes sistemas de recuperación del fallo adecuados (trato amable durante la resolución del fallo, reembolsos, retribuciones, descuentos, políticas flexibles) para evitar que interpongan una queja ante un tercero.

- En el sector servicios al contratar personal, se deben seleccionar candidatos con la habilidad para interactuar con otras personas, así como capacitar a los empleados en la atención de problemas o situaciones no previstas, haciendo énfasis en aspectos como no discutir con los clientes, tener un cierto rango de acción para tomar decisiones, y darle al cliente la razón, es decir, aplicar una política de marketing de servicios global.

- Prestar atención en el servicio a los clientes habituales. Muchas veces se hace un especial énfasis en programas de premios y bonos para clientes nuevos y se descuida el trato hacia los clientes habituales.

En resumen, si se pretenden evitar las quejas de los clientes, la empresa debe trabajar mucho en brindar el mejor trato (que el consumidor perciba amabilidad y cortesía en to do momento, independientemente del fallo), en conseguir la retención de clientes (un cliente habitual puede "perdonar" más un fallo que un cliente nuevo) y en proveer medios para que los clientes planteen sus quejas (oportunidades de mejora) directamente con la propia empresa.

\section{LIMITACIONES Y FUTURAS LÍNEAS DE INVESTIGACIÓN}

Como todo trabajo empírico, este estudio presenta una serie de limitaciones que podrían motivar futuras líneas de investigación. Sobre la muestra del estudio, se ha trabajado con una población homogénea (estudiantes universitarios). Sería interesante realizar el experimento con una población más heterogénea (estudiantes, empleados, pensionados) y comparar los resultados entre las distintas poblaciones. Además, se enriquecería el estudio al analizar una población de otro perfil, ta vez personas de negocios que constantemente se hospedan en hoteles, por el aporte de información que pueden ofrecer debido a una mayor experiencia tanto en la cantidad de encuentros como en el trato recibido.

Por otra parte, en cuanto al diseño del experimento, se podría plantear utilizar algún grupo de control, a quienes se les presentaría la misma encuesta, mostrándoles la misma situación de fallo, pero sin realizar manipulación de la experiencia previa ni de la justicia interpersonal. Además, para complementar la escala estudiada, podría presentarse alguna otra donde se profundice el tema del e-WOM y las quejas a través de Internet.

\section{REFERENCIAS}

Aguilar, O. y Fandos, C. (2013). El papel de la experiencia previa y la justicia interpersonal en el sistema de recuperación del servicio tras un fallo, Revista Española de Investigación de Marketing, 17 (2), 103-133. 
Alberca, P. y Parte, L. (2013). Evaluación de la eficiencia y la productividad en el sector hotelero español: un análisis regional. Investigaciones Europeas de Dirección y Economía de la Empresa, 19, 102-111.

Álvarez, A., Vila, M., Fraiz, J. y De la Cruz, M. (2013). Análisis de las relaciones de dependencia entre los factores críticos de la calidad y los resultados. Sector de alojamiento turístico en España. Investigaciones Europeas de Dirección y Economía de la Empresa, 19, 74-89.

Ambrose M., Hess R. y Ganesan S. (2007). The relationship between justice and attitudes: An examination of justice effects on event and systemrelated attitudes. Organizational Behavior and Human Decision Processes, 103 (1), 21-36.

Aquino, K. (1995). Relationships among pay inequity, perceptions of procedural justice, and organizational citizenship. Employee Responsibilities and Rights Journal, 8, 21-33.

Baer, J. (2011). Convince and convert: Digital Marketing Advisors. Recuperado de http://www. convinceandconvert.com/social-mediamonitoring/70-of-companies-ignore-customercomplaints-on-twitter

Baker, T., Meyer, T. y Chebat, J.C. (2013). Cultural impacts on felt and expressed emotions and third party complaint relationships. Journal of Business Research, 66, 816-822.

Bies, R. y Shapiro, D. (1987), Interactional fairness judgments: the influence of causal accounts. Social Justice Research, 1 (2), 199-218.

Blodgett, J., Hill, D. y Tax, S. (1997). The Effects of Distributive, Procedural, and Interactional Justice on Postcomplaint. Behavior. Journal of Retailing, 73 (2), 185-210.

Bolton, R., Grewal D., y Levy, M. (2007). Six strategies for competing through service: An agenda for future research. Journal of Retailing, 83, (1), 1-4.

Chadha, N. y Willigen, J. (1999). Social Aging in a Delhi Neighborhood. California: Praeger.

Chelminski, P. y Coulter, R. (2011). An examination of consumer advocacy and complaining behavior in the context of service failure. Journal of Services Marketing, 25 (5), 361-370.

Czepiel, J. (1990). Service Encounters and Service Relationships: Implications for Research. Journal of Business Research, 20 (1), 13-21.

Del Águila-Obra, A.R., Al-dweeri, R.M. y Padilla-Meléndez, A. (2012). Factores determinantes de la calidad de los servicios electrónicos en el contexto de los operadores postales. Universia Business Review, tercer trimestre, 114-123.

Dewitt T., Nguyen, D. y Marshall, R. (2008). Exploring Customer Loyalty Following Service Recovery: The Mediating Effects of Trust and Emotions. Journal of Service Research, 10 (3), 269-281.

Eagly, A. (1987). Las diferencias de sexo en el comportamiento social: Una interpretación de rol social. Hillsdale, Nueva Jersey: Erlbaum.

Eagly, A., Wood, W. y Diekman, A. (2000). La teoría del rol social de las diferencias y similitudes sexuales: Una evaluación actual, en T. Eckes y HM Trautner Eds., La psicología del desarrollo social del género. Mahwah, NJ: Erlbaum.

Europa Press. (2011). Naciones Unidas declara el acceso a Internet como un derecho humano, periódico El Economista.es, 9 de setiembre del 2011. http://www.eleconomista.es/tecnologia-internet/noticias/3141485/06/11/La-ONU-declara-elacceso-a-Internet-como-un-derecho-humano. html

Ferguson, J. y Johnston, W. (2011). Customer response to dissatisfaction: A synthesis of literature and conceptual framework. Industrial Marketing Management, 40, 118-127.

Folger, R. (1987). Reformulating the preconditions of resentment: A referent cognitions model. En J. C. Masters y W. Smith Eds., Social comparison, justice, and relative deprivation: Theoretical, empirical, and policy perspectives. Hillsdale, NJ: Erlbaum.

González, E., Alén, M.E. y Rodríguez, L. (2011). Benchmarking en responsabilidad social corporativa: el caso de las empresas gallegas. Cuadernos de Gestión, 11, Especial Responsabilidad Social, 101-119. 
Han H., Hsu, L. y Lee, J. (2009). Empirical investigation of the roles of attitudes toward green behaviors, overall image, gender, and age in hotel customers' eco-friendly decision-making process. International Journal of Hospitality Management, 28, 519-528.

Han, S., Keng, K.A. y Richmond, D. (1995). Determinants of consumer complaint behavior: a study of Singapore consumers. Journal of International Consumer Marketing, 8 (2), 59-76.

Hannigan, J. (1977). The Newspaper Ombudsman and Consumer Complaints. Empirical Assessment, 11 (4), 679-699.

Henry, A. y Ho, D. (2010). The act of complaining in Brunei-Then and now. Journal of Pragmatics, 42, 840-855.

Hernández, B., Jiménez, J. y Martín, M. (2011). Age, gender and income: do they really moderate online shopping behaviour? Online Information Review, $35(1), 113-133$.

Heung, V. y Lam, T. (2003). Customer complaint behaviour towards hotel restaurant services. International Journal of Contemporary Hospitality Management, 5 (5), 283-289.

Hogarth, J., English, M. y Sharma, M. (2001). Consumer complaints and third parties: determinants of consumer satisfaction with complaint resolution efforts. Journal of Consumer Satisfaction, Dissatisfaction and Complaining Behavior, 14, 74-87.

Homburg, C. y Giering, A. (2001). Personal characteristics as moderators of the relationship between customer satisfaction and loyalty: an empirical analysis. Psychology and Marketing, 18 (1), 43-66.

Honeycutt, E., Magnini, V. y Thelen, S. (2012). Solutions for customer complaints about offshoring and outsourcing services. Business Horizons, 55, 33-42.

Hosteltur. (2013). Hosteltur. Calidad y servicio claves en la competitividad hotelera española. Recuperado de: http://www.hosteltur.com/150290_calidad-servicio-claves-competitividad-hotelera-española.html.

Hui, M., Au, K. y Zhao, X. (2007). Interactional justice and the fair process effect:The role of outcome uncer- tainty. Journal of Experimental Social Psychology, 43, 210-220.

lacobucci, D. y Ostrom, A. (1993). Gender differences ir the impact of core and relational aspects of services on the evaluation of service encounters. Journal of Consumer Psychology, 2, 257-286.

IET. (2016). Balance del turismo, año 2012: Resultados de la actividad turística en España, Ministerio de Industria, Energía y Turismo, Instituto de Estudios Turísticos. Recuperado de: http://estadisticas.tourspain. es/es-ES/estadisticas/analisisturistico/balantur/ anuales/Balance\%20del\%20turismo\%20en\%20 Espa\%C3\%B1a.\%20A\%C3\%B10\%202012.pdf

INC. (2014). Balance de consultas y reclamaciones presentadas en las organizaciones de consumidores de ámbito nacional: año 2013. Ministerio de Sanidad, Política Social e Igualdad, Instituto Nacional de Consumo. http://consumo-inc.gob.es/memorias/ MEMORIAINC2013.pdf

Kalamas, M., Laroche, M. y Makdessian, L. (2008). Reaching the boiling point: consumers' negative affective reactions to firm-attributed service failures. Journal of Business Research, 61(8), 813-24

Kim, B., Choi, M. y Han, I. (2009). User behaviors toward mobile data services: The role of perceived fee and prior experience. Expert Systems with Applications, 36, 8528-8536.

Kitapci, O. y Dortyol, I. (2009). The differences in customer complaint behaviour between loyal customers and first comers in the retail banking industry: The case of Turkish customers. Management Research News, 32 (10), 932-941.

Laguna, M. y Palacios, A. (2009). La calidad percibida como determinante de tipologías de clientes y su relación con la satisfacción: Aplicación a los servicios hoteleros. Revista Europea de Dirección y Economía de la Empresa, 18 (3), 189-212.

Laurent, G. y Kapferer, J. (1985). Measuring Consumer Involvement Profiles. Journal of Marketing Research. 22 (February): 41-53. 
Lerman, D. (2006). Consumer politeness and complaining behavior. Journal of Services Marketing, 20 (2), 92-100.

Levinson, D.J. (1986). A conception of adult development. American Psychologist, 41 (3), 3-13.

Liu, R. y McClure, P. (2001). Recognizing cross-cultural differences in consumer complaint behavior and intentions: an empirical examination. Journal of Consumer Marketing, 18 (1), 54-74.

Mattila, A. (2010). Do women like options more than men? An examination in the context of service recovery. Journal of Services Marketing, 24 (7), 499-508.

McAlister, D. y Erffmeyer, R. (2003). A content analysis of outcomes and responsibilities for consumer complaints to third-party organizations. Journal of Business Research, 56, 341-351.

McColl-Kennedy, J. y Sparks, B. (2003). Application of fairness theory to service failures and service recovery. Journal of Service Research, 5 (3), 251-266.

McColl-Kennedy, J., Daus, C. y Sparks, B. (2003). The role of gender in reactions to service failure and recovery. Journal of Service Research, 6 (1), 66-81.

Méndez, A. (27 de febrero de 2013). "Saca partido del negocio de la queja", Periódico Expansión. com. recuperado de: http://www.expansion. com/2013/02/26/emprendedores-empleo/emprendimiento/1361901894.html

Moliner, B. y Fuentes, M. (2011). Causas y consecuencias de la insatisfacción en consumidores con atribuciones externas. Cuadernos de Gestión, 11 (1), 37-58.

Moliner B., Fuentes, M., Gil, I. y Berenguer, G. (2010). Causes for complaining behaviour intentions: the moderator effect of previous customer experience of the restaurant. Journal of Services Marketing, 24 (7), 532-545.

Moliner, B., Berenger, G., Gil, I. y Fuentes, M. (2008). Análisis del comportamiento de queja del consumidor: una investigación exploratoria en el contexto de los restaurantes. Investigaciones Eu- ropeas de Dirección y Economía de la Empresa, 14 (2), 13-33.

Moschis, G., (1994). Consumer behavior in later life: multidisciplinary contributions and implications for research. Journal of the Academy of Marketing Science, 22 (3), 195-204.

Namkung, Y., Jang, S. y Choi, S. (2011). Customer complaints in restaurants: Do they differ by service stages and loyalty levels? International Journal of Hospitality Management, 30, 495-502.

Namkung, Y. y Jang, S. (2009). The effects of interactional fairness on satisfaction and behavioral intentions: Mature versus non-mature customers. International Journal of Hospitality Management, 28, 397-405.

Nunnally, J. (1978). Psychometric Theory. New York: McGraw-Hill.

Ok, C., Back, K. y Shanklin, C. (2005). Modeling roles of service recovery strategy: a relationship-focused view. Journal of Hospitality and Tourism Research, 29 (4), 484-507.

Parte, L. y Such, M. (2011). Earnings benchmarks en el sector hotelero: decisiones basadas en variables financieras y reales. Investigaciones Europeas de Dirección y Economía de la Empresa, 17 (1), 113135.

Phillips, L.W. y Sternthal, B., (1977). Age differences in information processing: a perspective on the aged consumer. Journal of Marketing Research, $14,444-457$.

Rawls, J. (1977). A theory of Justice. Harvard University Press.

Reiboldt, W. (2003). Factors that influence a consumer complainer's rating of service received from a third party complaint-handling agency - the Los Angeles Department of Consumer Affairs. Journal of Consumer Satisfaction, Dissatisfaction and Complaining Behavior, 16, 166-77.

Riley, M., Johnson, M. y Foner, A. (1972). Aging and Society. Volume III, A Sociology of Age Stratification. New York: Russell Sage Foundation. 
Ruyter, K. y Wetzels, M. (2000). The impact of perceived listening behavior in voice-to voice service encounters. Journal of Service Research, 2 (3), 276284.

Ryngelblum, A., Vianna, N. y Rimoli, C. (2013). The ways companies really answer consumer complaints, Marketing Intelligence and Planning, 31 (1), 54-71.

Sampedro, J. (12 de setiembre 2012). Un mensaje en Facebook movilizó 340.000 votos en un solo día, periódico El País. Recuperado de: http://sociedad.el pais.com/sociedad/2012/09/12/actualidad/1347463613_247798.html

San Martín, H., Collado, J., y Rodríguez, I. (2008). El proceso global de satisfacción bajo múltiples estándares de comparación: el papel moderador de la familiaridad, la involucración y la interacción cliente-servicio. Revista Española de Investigación de Marketing, ESIC, 12(1), 65-95.

Schoefer, K. y Diamantopoulos, A. (2008). The Role of Emotions in Translating Perceptions of InJustice into Postcomplaint Behavioral Responses. Journal of Service Research, 11 (1), 91-103.

Shapiro, T. y Nieman-Gonder, J., (2006). Effect of communication mode in justice-based service recovery. Managing Service Quality, 16 (2), 124-144.

Simcock, P., Sudbury, L. y Wright, G., (2006). Age, perceived risk and satisfaction in consumer decision making: a review and extension. Journal of Marketing Management, 22 (3/4), 355-377.

Singh, J. (1988). Consumer complaint intentions and behavior: definitional and taxonomical issues. Journal of Marketing, 1, 94-107.

Tax, S.S., Brown, S.W. y Chandrashekaran, M. (1998). Customer evaluations of service complaint experiences: implications for relationship marketing. Journal of Marketing, 62 (2), 60-76.

Tipper, R. (1997). Characteristics of consumers who seek third party redress. Consumer Interests Annual, 4, 222-226.
Tongren, H.N., (1988). Determinant behavior characteristics of older consumers. Journal of Consumer Affairs, 22 (1), 136-157.

Van Noort, G. y Willemsen, L. (2011). Online Damage Control: The Effects of Proactive Versus Reactive Webcare Interventions in Consumer-generated and Brand-generated Platforms. Journal of Interactive Marketing, 26, 131-140.

Vásquez, C. (2011). Complaints online: The case of TripAdvisor. Journal of Pragmatics, 43, 1707-1717.

Vázquez-Casielles R., del Río-Lanza, A. B. y SuárezÁlvarez, L. (2009). Las agencias de viaje virtuales: ¿Cómo analizar la calidad de e-servicio y sus efectos sobre la satisfacción del cliente? Universia Business Review, cuarto trimestre, 122-142.

Wu, I. (2013). The antecedents of customer satisfaction and its link to complaint intentions in online shopping: An integration of justice, technology, and trust. International Journal of Information Management, 33, 166-176.

Yelkur, R. y Chakrabarty, S. (2006). Gender differences ir service quality expectations in the fast food Industry. Services Marketing Quarterly, 27 (4), 141-151.

Yuksel A., Kilinc, U. y Yuksel, F. (2006). Cross-national analysis of hotel customers' attitudes toward complaining and their complaining behaviours. Tourism Management, 27, 11-24. 
This document was created with Win2PDF available at http://www.win2pdf.com. The unregistered version of Win2PDF is for evaluation or non-commercial use only. This page will not be added after purchasing Win2PDF. 